\title{
Intrathecal Colistin and Intravenous Fosfomycin as a Combination Therapy for the Treatment of Acinetobacter Baumannii Ventriculitis and Meningitis
}

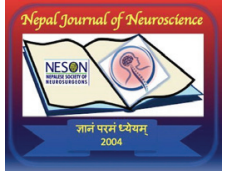

Bikash Khadka $\mathbf{M D}^{1}$ (D), Saroj Poudel PharmD ${ }^{2}$ (D)

${ }^{1}$ Department of Anesthesiology and Critical Care, Nepal Mediciti, Lalitpur, Nepal

${ }^{2}$ Clinical Pharmacist, Nepal Mediciti, Lalitpur, Nepal

Date of submission: $6^{\text {th }}$ June 2021

Date of acceptance: $22^{\text {nd }}$ October 2021

Date of publication: $1^{\text {st }}$ December 2021

\section{Abstract}

Treatment of central nervous system infection may be troublesome due to multidrug resistance. Colistin is less successful as a treatment option due to poor central nervous system penetration when used intravenously. We present the successful management of a case with ventriculitis and meningitis due to multidrug resistant Acinetobacter baumannii species with the combined intraventricular administration of colistin and intravenous fosfomycin after the initial regimen of colistin given alone through both intravenous and intrathecal routes had failed.

Key words: Acinetobacter baumannii, Colistin, Fosfomycin, Intrathecal, Multidrug resistant.

\section{Introduction}

Central nervous system (CNS) infections such as meningitis, ventriculitis, empyema and brain abscesses are extremely serious and difficult to treat. Neurosurgical interventions (craniotomy or ventriculostomy placement), craniocerebral trauma and invasive neuromonitoring techniques may also cause CNS infections. A recent retrospective study found an overall meningitis rate of $1.52 \%$ in 6,243 subjects undergoing craniotomy. ${ }^{1} \mathrm{CNS}$ infections necessitate immediate and well-targeted treatment as it has high risk of neurological and systemic complications.

\section{Access this article online}

Website: https://www.nepjol.info/index.php/NJN

DOI: https://doi.org/10.3126/njn.v18i4.37518

HOW TO CITE

Khadka B, Poudel S. Intrathecal Colistin and Intravenous Fosfomycin as a Combination Therapy for the Treatment of Acinetobacter Baumannii Ventriculitis and Meningitis. NJNS. 2021;18(4):39-43.

\section{Address for correspondence: \\ Dr. Bikash Khadka \\ Nepal Mediciti, \\ Bhainsepati, Lalitpur, Nepal. \\ E-mail:khadka.vkas@gmail.com \\ Phone: +977 9841411827}

Copyright (C) 2021 Nepalese Society of Neurosurgeons (NESON)

ISSN: 1813-1948 (Print), 1813-1956 (Online)
Acinetobacter baumannii is a gram-negative coccobacillus that can cause bacteremia, pneumonia, urinary tract infections and surgical wound infections. Meningitis is a rare but serious condition caused by acinetobacter infection, most commonly seen following the insertion of an indwelling ventriculostomy catheter, cerebrospinal fluid (CSF) leakage or head trauma. CNS infection associated with Acinetobacter baumannii has an incidence of 3.6 to $11 \%{ }^{2}$

Physical barriers and physicochemical properties of the antimicrobial agents have an influence on the drug distribution into the CNS. The tight junctions within the blood brain barrier (BBB) and blood-CSF barrier limit the diffusion of large molecular weight substances (500 Daltons). Antimicrobial agents with larger molecular weights (e.g. vancomycin or amphotericin B) have limited ability to penetrate an intact barrier. The $\mathrm{pH}$ of blood, CSF and extracellular fluid can change with infection and this can influence the polarity and hence anti-infective penetration of the CNS. ${ }^{3}$ Antibiotics such as aminoglycosides, glycopeptides and polymyxins have a very low therapeutic index with low CSF concentrations and therefore require lumbar, intrathecal (IT) or intraventricular (IVT) administration. ${ }^{4}$ The ventricles can act as a persistent reservoir of infection and inflammation, resulting in extreme difficulty in eradicating infection and potential blockade of CSF outflow tracts. ${ }^{5}$ Intraventricular and intrathecal administration of antibiotics may be necessary to overcome these difficulties.

We present a case of multiple drug resistant Acinetobacter baumannii post surgical meningitis that was successfully treated with high doses of fosfomycin combined with intrathecal colistin. 


\section{Case report}

A 22-year-old man was admitted to the intensive care unit of our institute with a serious injury to his face and head with initial loss of consciousness following a bike accident. On neurological examination, he had a wound over the forehead with CSF. The cranial and cervical computed tomography (CT) scans revealed bifrontal contusion, a left temporal intracerebral hematoma with findings shown in figure1 below. The patient underwent immediate decompression craniectomy and repair of the anterior cranial fossa. The patient was empirically treated with ceftriaxone (1g IV every $12 \mathrm{~h}$ ) and vancomycin (1g IV every $12 \mathrm{~h}$ ) because of high total lymphocyte counts (TLC of 14180) and persistent fever $\left(38.3^{\circ} \mathrm{C}\right)$.

Due to persistent fever and signs and symptoms of raised intracranial pressure on $10^{\text {th }}$ post-operative day, external ventricular drainage (EVD) was inserted and CSF analysis was done. CSF analysis showed it was turbid in appearance, watery, WBC count of 280 cells/mm3 (polymorphonuclear neutrophils $95 \%$, lymphocytes $5 \%$ ), glucose level $<20 \mathrm{mmol} / \mathrm{l}$, protein 3 $\mathrm{g} / 1$ and Acinetobacter baumannii organism growth in culture. Antimicrobial susceptibility showed the strain was resistant to cephalosporins, beta-lactam, carbapenem and aminoglycosides but susceptible to tigecycline, colistin, Polymyxin-B (PMB) and ofloxacin. Following multidisciplinary team discussion, the patient was put under intraventricular colistin via EVD because of low CSF penetration of parenterally administered tigecycline and PMB. ( colistin methanesulfonate (CMS) dosing: $10 \mathrm{mg}$ IVT per day and EVD clamp for 2 hours post colistin administration). Note: 1 vial $=1$ million units $=80 \mathrm{mg}(1$ vial diluted with $8 \mathrm{ml} 0.9 \%$ normal saline $=1 \mathrm{ml} / 10 \mathrm{mg}$ ). Before CMS administration, $5 \mathrm{ml}$ of CSF was aspirated and discarded (to avoid an increase of intracranial pressure induced by the bolus of CMS). After CMS administration, the ventricular drainage was flushed immediately with $2 \mathrm{ml}$ of saline solution to minimize the dose remaining in the drainage. The CMS was injected and the CSF samples were collected under sterile conditions and disinfected with chlorhexidine. The EVD was kept closed for at least 60-120 minutes.

On the 14th postoperative day, CSF analysis was repeated which showed turbid appearance, WBC count of 318 cells/mm3 and growth of Acinetobacter baumannii. Antimicrobial susceptibility was determined and the strain was now resistant to ofloxacin which was stopped and sulfamethoxazole/trimethoprim was added as per susceptibility.

Even after 7 days (17 th day of illness) of intraventricular colistin treatment, the patient's clinical status did not improve. Repeat CSF analysis showed turbid appearance, WBC count of 5800 cells $/ \mathrm{mm}^{3}$ and
Acinetobacter baumannii growth in culture. We planned for parenteral CMS $\{9$ million units loading dose followed by 3 million units every 8 hours (As CSF penetration of CMS is more than 25\%) $\}$ administration in place of IVT. As colistin monotherapy shows treatment failure, a high dose of parenteral Chloramphenicol ( 1 g every $6 \mathrm{~h}$ ) was considered.

On the 27th day of illness, 10 days following IV CMS, 14 days for oral sulfamethoxazole/trimethoprim and 5 days for IV chloramphenicol, CSF culture was repeated which again had growth of Acinetobacter baumanni as shown in table below. Chloramphenicol was stopped and as a salvage therapy for MDR gram-negative and grampositive organism, IV fosfomycin was added following multi-disciplinary team discussion ( $4 \mathrm{~g}$ of IV fosfomycin as a loading dose followed by $4 \mathrm{~g}$ every 6 hours). As IV fosfomycin has been reported to cause electrolyte disturbance (Hypernatremia and hyperkalemia) dilution was made in D5W (4 $\mathrm{g}$ of fosfomycin was dissolved in $100 \mathrm{ml}$ D5W and infused over 4 hour's interval).

After 48 hours of intravenous administration of fosfomycin (Day 29), persistent fever subsided and patient was hemodynamically stable. After 5 days of intravenous administration of fosfomycin, a repeated CSF culture was sent and a negative result was found (no organism was isolated in CSF). IV fosfomycin was continued for 5 more days. No adverse effects were observed during antibiotic therapy. Treatment for lower respiratory tract infection was continued in ICU for another week and was later planned for Lumboperitoneal shunt placement.

\section{Discussion}

Acinetobacter baumanii is responsible for $10 \%$ of all gram-negative meningitis and $4 \%$ of nosocomial meningitis. ${ }^{12}$ The high mortality rates observed in patients with A. baumanii meningitis can be attributed in part to therapeutic difficulties associated with frequent inadequate antibiotic therapy and increased bacterial resistance due to which infrequently used antibiotics have spurred into the markets like fosfomycin and Polymyxins. ${ }^{6}$

\section{Colistin}

Intraventricular colistin administered as CMS (colistin methanesulfonate) is used for the treatment of CNS infections caused by pan-resistant gram-negative bacteria. Intravenous colistin alone does not provide CSF concentrations high enough to reach a MIC of $2 \mathrm{mcg} / \mathrm{mL}$ for multidrug resistant (MDR) Gram-negative rods and therefore topical administration is needed to support the treatment. ${ }^{6}$ Colistin diffusion from the CSF to the systemic circulation and the cerebral tissue and the elimination through the external efflux of CSF, in addition to the unpredictable amount of the CSF spontaneously drained 
Intrathecal Colistin and Fosfomycin for CNS Infection

\begin{tabular}{|c|c|c|c|c|c|}
\hline Characters/Day & $16^{\text {th }}$ POD & $27^{\text {th }}$ POD & $34^{\text {th }}$ POD & $40^{\text {th }}$ POD & $47^{\text {th }}$ POD \\
\hline Colour/ appearance & $\begin{array}{c}\text { Yellowish/ Slight } \\
\text { turbid }\end{array}$ & Reddish/ Turbid & $\begin{array}{l}\text { Light } \\
\text { Yellow }\end{array}$ & Light yellow & Light yellow \\
\hline TC(cells/cumm) & 28600 & 5800 & 3280 & 65 & 200 \\
\hline Neutrophil (\%) & 98 & 100 & 93 & 00 & 5 \\
\hline Lymphocyte (\%) & 2 & 00 & 7 & 100 & 95 \\
\hline Glucose(mg/dl) & $<20$ & $<20$ & $<20$ & 44 & 62 \\
\hline Protein(mg/dl) & 714 & 495 & 594 & 373 & 565 \\
\hline RBC(cells/cumm) & 27000 & 12800 & 1350 & 400 & 100 \\
\hline Culture & $\begin{array}{c}\text { Acinetobacter } \\
\text { baumannii isolated }\end{array}$ & $\begin{array}{c}\text { Acinetobacter } \\
\text { baumannii isolated }\end{array}$ & $\begin{array}{c}\text { Acinetobacter } \\
\text { baumannii isolated }\end{array}$ & NO growth & NO growth \\
\hline
\end{tabular}

Table 1: Serial CSF analysis findings. POD: post operative day TC: Total count RBC: red blood cells.
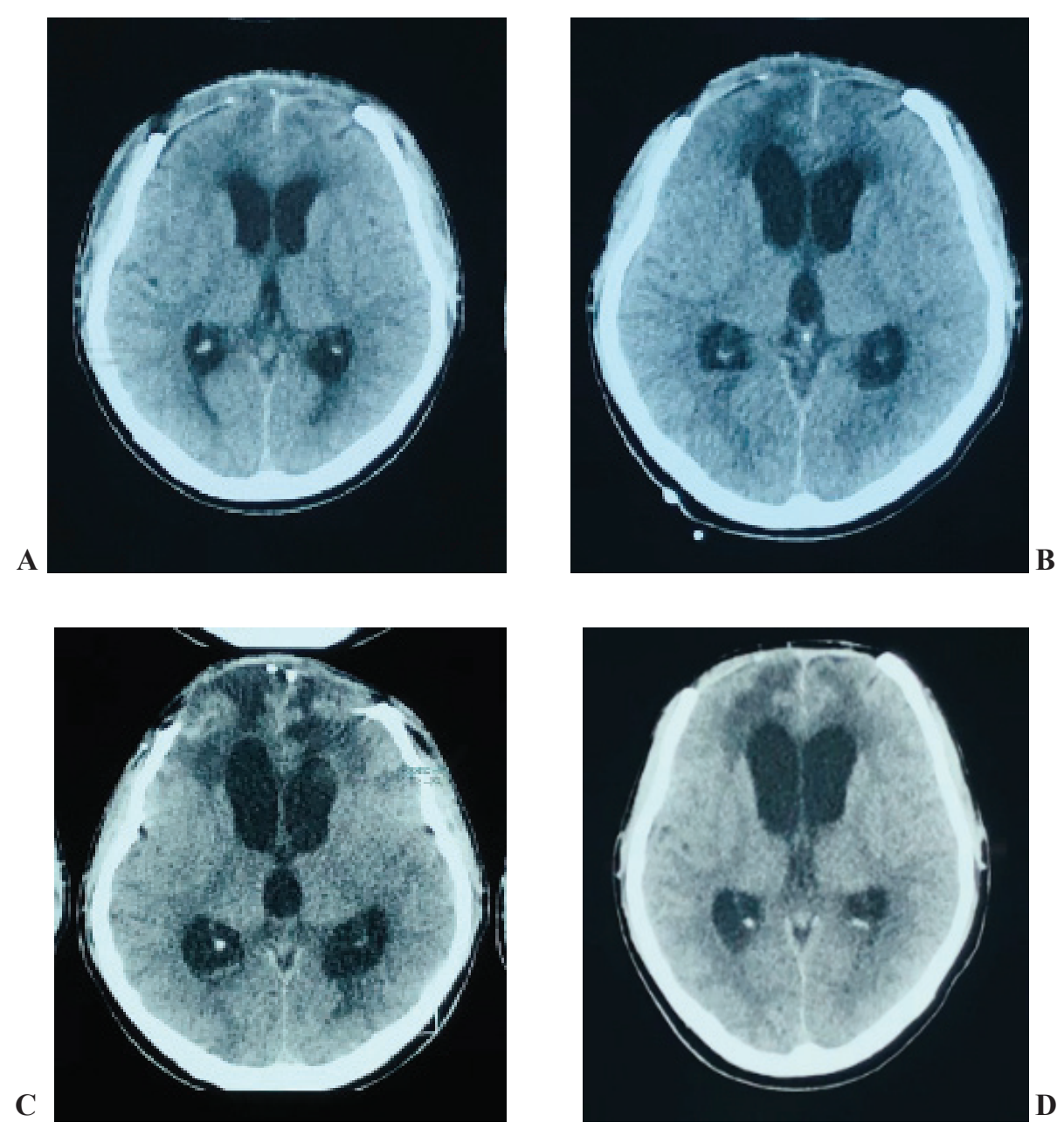

Figure 1: Serial CT Scan head finding: (A) Post central frontal craniectomy with scalp swelling, with mild herniation of frontal lobe and minimal SDH. (B) Post craniectomy with pneumocephalus in right frontal lobe with EVD in situ, mild dilatation of lateral and third ventricles (after a week of Scan A) with the start of intravenous colistin and 5 days of intrathecal Colistin administration. (C) Post craniectomy status with dilated ventricles (after 12 days of Scan A) on iv colistin and before start of intravenous Fosfomycin. (D) Post craniectomy status with reduced mass effect and herniation of frontal lobe (after 4 weeks of Scan A), corresponding to completion of treatment of IV Fosfomycin. 


\section{Khadka et al}

through the EVD by the fluctuation of intracranial pressure showing its appropriateness for treatment and IT/IVT dose of 125,000 IU/day suggested by the IDSA guidelines, but optimal IT/IVT dose remains uncertain. ${ }^{7}$

\section{Fosfomycin}

Fosfomycin (FOF) is a low molecular weight antibiotic that has bactericidal activity against both Gram-negative and Gram-positive bacteria and reported that minimum inhibitory concentration (MIC) against $A$. baumannii is very high. The administration of intravenous fosfomycin has recently been revised due to its favorable pharmacokinetic properties, high tissue penetration, even in deep-seated infections, and low toxicity. Because the permeability of cerebrospinal fluid increases when the meninges become inflamed, the concentration of fosfomycin in the CSF can nearly triple in the case of meningitis. $^{8}$

Carbapenems have excellent in vitro activity against Acinetobacter baumannii and have been shown to have significant clinical success rates in the treatment of A. baumannii meningitis. As a result, antimicrobial options are extremely limited. Polymyxins are often the only antibiotics that show in vitro activity against multi-resistant strains. Colistin is an old drug that was abandoned decades ago due to nephrotoxicity. Although its permeability to cerebrospinal fluid is poor, it has recently become a new option or gained interest for the treatment of multidrugresistant Acinetobacter baumannii.

In these cases, the identification of synergisms between antibiotics is an important tool, both due to the lack of treatment options and the recommended combination therapy with certain antibiotics such as fosfomycin and polymyxins, to achieve a more effective therapeutic response. ${ }^{9}$

There are guidelines available such as those by the Infectious Diseases Society of America (IDSA) in 2004 that suggested a daily IT dosage of $10 \mathrm{mg}(125,000$ IU). ${ }^{9}$ We also followed the same guideline for colistin administration. A cumulative dose above 1,750,000 IU of IT/IVT colistin (which corresponds to $125,000 \mathrm{IU}$ once daily for 14 days) is associated with better outcomes. ${ }^{6}$ We administered for a total duration of 14 days but it was with no success. The duration of therapy described is quite variable, from one to four weeks. ${ }^{6}$ We opted to use intrathecal colistin until the first CSF culture growth report came negative.

Astudy by Imbertietal.describing the pharmacokinetics of IT/IVT colistin with dosages, from 2.61 to $10.44 \mathrm{mg}$ (32,000-130,000 IU) showed CSF concentration of $>2 \mathrm{mcg} / \mathrm{mL}$ when colistin was administered at a dose $>65,200 \mathrm{IU} /$ day, with great inter-patient variability. ${ }^{10}$ Twelve patients treated with IVT-IV colistin $(3,000,000$ IU every 8 hours and IVT dose of 125,000 IU daily) and EVD were studied by HPLC by Ziaka, of which five were treated only IV, three only by IVT administration, and four patients by IVT-IV. ${ }^{11}$ The CSF concentrations in the IV group were very low, with a ratio of $0.07 .{ }^{11}$ There was no accumulation of colistin concentration over time, the mean $\mathrm{CSF} /$ serum concentration was higher in the Intraventricular-IV combination than in the other groups and the mean AUC CSF/AUC serum ratios were $60 \%$ higher in patients with ventriculitis than in control patients. Perhaps a greater penetration could be achieved with meningeal inflammation but a median CSF concentration $>0.5 \mathrm{mcg} / \mathrm{mL}$ was only observed in the combination group, suggesting that IV colistin could increase CSF concentrations of IT/Intraventricular colistin, the conclusion was that combined treatment had a better chance to eradicate severe CNS infections. ${ }^{14}$

A study by Tumbarello $M$ suggested that in ventriculitis, IV colistin should be added to IT/IVT colistin and perhaps a second systemic antimicrobial, chosen according to the susceptibility and synergy tests. ${ }^{11}$ Since clinical improvement was not seen we started the patient on IV fosfomycin for a synergistic effect. In vitro and in vivo studies suggest that fosfomycin acts in synergy with other antimicrobial agents, such as polymyxins and carbapenems. ${ }^{13}$ Clinical improvement was seen within 48 hours with subsiding fever, improved orientation, hemodynamic stability, and negative culture growth on subsequent CSF analysis. There were no significant signs of toxicity, clinical and laboratory data. The antibiotic CSF concentrations that would have allowed comparisons with the MIC's of the isolates were not measured which could have helped analyze it much better.

The combinations of FOF + meropenem and FOF + Polymyxin B had the lowest rates of synergism, with only one isolate of acinetobacter. PMB-resistant Acinetobacter isolates displayed a reduction in the MICs of PMB and FOF and were classified as susceptible (MICs of $2 \mathrm{~g} / \mathrm{mL}$ and $32 \mathrm{~g} / \mathrm{mL}$, respectively). ${ }^{14}$ FOF showed good results when combined with other antimicrobials; however, care should be taken with its use as monotherapy due to the rapid in vitro emergence of resistance in addition to the selection of resistant microorganisms.

A recent in vivo study using FOF combined with colistin found significantly higher microbial elimination over $72 \mathrm{~h}$ of treatment in the group of patients treated with a combination of the two drugs $(90.7 \%)$ than in the group treated with colistin alone $(58.1 \%) .{ }^{16}$ At the end of treatment, complete microbiological elimination occurred in $100 \%$ of patients receiving the combination therapy compared with only $81.2 \%$ of patients treated with colistin alone. ${ }^{16}$

Fosfomycin has been reported to show a $100 \%$ synergistic effect after combining with other antibacterial drugs. ${ }^{15}$ Fosfomycin prevents the first stage of the cell wall synthesis procedure, whereas other antibiotics (such as carbapenem, polymyxins) inhibit the final phase. 


\section{Conclusion}

For multidrug resistant meningitis and ventriculitis, intraventricular colistin along with iv fosfomycin has proved to be effective and safe. This combination, compared to the single colistin intraventricular administration, resulted in quick microbiologic and clinical improvement and future studies may refine further knowledge about the safety of this "salvage treatment".

\section{Acknowledgment}

I would like to thank Dr. Diptesh Aryal, ICU coordinator for his constant support and guidance.

Conflict of Interest: None

Source(s) of support: None

\section{References}

1. Korinek AM, Reina M, Boch AL, Rivera AO, De Bels D, Puybasset L. Prevention of external ventricular drain--related ventriculitis. Acta Neurochir (Wien). 2005;147(1):39-45; discussion -6.

2. Kim BN, Peleg AY, Lodise TP, Lipman J, Li J, Nation $\mathrm{R}$, et al. Management of meningitis due to antibioticresistant Acinetobacter species. Lancet Infect Dis. 2009;9(4):245-55. https://doi.org/10.1016/s14733099(09)70055-6

3. Kearney BP, Aweeka FT. The penetration of antiinfectives into the central nervous system. Neurol Clin. 1999;17(4):883

4. Bargiacchi O, De Rosa FG. Intrathecal or intraventricular colistin: a review. Infez Med. 2016;24(1):3-11

5. Salmon JH. Ventriculitis complicating meningitis. Am J Dis Child. 1972;124(1):35-40.

6. Falagas ME, Kastoris AC, Karageorgopoulos DE, Rafailidis PI. Fosfomycin for the treatment of infections caused by multidrug-resistant nonfermenting Gramnegative bacilli: a systematic review of microbiological, animal and clinical studies. Int J Antimicrob Agents 2009;34:111-20. https://doi. org/10.1016/j.ijantimicag.2009.03.009

7. Tunkel AR, Hartman BJ, Kaplan SL, Kaufman BA, Roos KL, Scheld WM, et al. Practice guidelines for the management of bacterial meningitis. Clin Infect Dis. 2004;39(9):1267-84. https://doi.org/10.1086/425368
8. Kühnen E, Pfeifer G, Frenkel C. Penetration of fosfomycin into cerebrospinal fluid across noninflamed and inflamed meninges. Infection. 1987;15(6):422-4. https://doi.org/10.1007/ bf01647220

9. Dubrovskaya Y, Chen T, Scipione MR, Esaian D, Phillips MS, Papadopoulos, et al. Risk factors for treatment failure of polymyxin B monotherapy for carbapenem-resistant Klebsiella pneumoniae infections. Antimicrob Agents Chemother 2013;57:5394-7. https://doi.org/10.1128/aac.0051013

10. Imberti R, Cusato M, Accetta G, Marinò V, Procaccio F, Del Gaudio A, et al. Pharmacokinetics of colistin in cerebrospinal fluid after intraventricular administration of colistin methanesulfonate. Antimicrob Agents Chemother. 2012;56(8):4416-21. https://doi.org/10.1128/aac.00231-12

11. Ziaka M, Markantonis SL, Fousteri M, Zygoulis $\mathrm{P}$, Panidis D, Karvouniaris $\mathrm{M}$, et al. Combined intravenous and intraventricular administration of colistin methanesulfonate in critically ill patients with central nervous system infection. Antimicrob Agents Chemother. 2013;57(4):1938-40. https://doi. org/10.1128/aac.01461-12

12. Tumbarello M, Viale P, Viscoli C, Trecarichi EM, Tumietto F, Marchese A, et al. Predictors of mortality in bloodstream infections caused by Klebsiella pneumoniae carbapenemase-producing K. pneumoniae: importance of combination therapy. Clin Infect Dis. 2012;55(7):943-50. https://doi. org $/ 10.1093 / \mathrm{cid} / \mathrm{cis} 588$

13. Sirijatuphat R, Thamlikitkul V. Preliminary study of colistin versus colistin plus fosfomycin for treatment of carbapenem-resistant Acinetobacter baumannii infections. Antimicrob Agents Chemother 2014;58:5598-601 https://doi.org/10.1128/ aac. $02435-13$

14. Menegucci TC, Albiero J, Migliorini LB, Alves JL, Viana GF, Mazucheli J, et al. Strategies for the treatment of polymyxin B-resistant Acinetobacter baumannii infections. Int $\mathrm{J}$ Antimicrob Agents. 2016;47(5):380-5. https://doi.org/10.1016/j. ijantimicag.2016.02.007

15. Hashemian SMR, Farhadi Z, Farhadi T. Fosfomycin: the characteristics, activity, and use in critical care. Ther Clin Risk Manag. 2019;15:525-530. https://doi.org/10.2147/TCRM.S199119 